(C2021 IEEE. Personal use of this material is permitted. Permission from IEEE must be obtained for all other uses, in any current or future media, including reprinting/republishing this material for advertising or promotional purposes, creating new collective works, for resale or redistribution to servers or lists, or reuse of any copyrighted component of this work in other works. This is the author's version of an article that has been accepted for publication in the conference proceedings. 


\title{
Radio Interference Measurements for Urban Cooperative Intelligent Transportation Systems
}

\author{
Stephan Sand, Paul Unterhuber, Dina Bousdar Ahmed, Fabian de Ponte Müller, Andreas Lehner, Ibrahim Rashdan, \\ Martin Schmidhammer, Rostislav Karásek, Benjamin Siebler, Oliver Heirich, Christian Gentner, Michael Walter, \\ Susanna Kaiser, Markus Ulmschneider, Marius Schaab, Luis Wientgens, Thomas Strang \\ German Aerospace Center (DLR), Institute of Communications and Navigation, \\ Oberpfaffenhofen, 82234 Wessling, Germany, stephan.sand@dlr.de
}

\begin{abstract}
The trend towards urbanization increases the need for highly available public transportation. Nevertheless, the majority of society demands for individual transport too. To address these demands in urban areas, intelligent transportation systems (ITS) aim at increasing capacity and safety while reducing costs, accidents, and environmental impact. To that end, both the railway industry and the automotive industry focus on automation, digitization, and wireless communications to cope with increasing numbers of vehicles and passengers. These two industries may rely on cooperative ITS (C-ITS) communicating in the same frequency band. Without appropriate measures, interference between the different radio technologies must be assumed and reliable communication for safety-critical applications cannot be guaranteed. To develop accurate and realistic interference models for current and future radio technologies, we conducted a four-day measurement campaign with the Deutsche Bahn (DB) advanced TrainLab on the Berlin "Süd-Ring" tracks. In this paper, we present an overview on C-ITS radio technologies, the measurement campaign, first results, and conclusions. An initial data analysis shows that adjacent channel interference can cause severe performance degradation on urban rail C-ITS if generated in line-of-sight (LOS) to the train with a significant number of interfering signals.

Index Terms-C-ITS, ITS-G5, LTE C-V2X, IEEE 802.11a,
\end{abstract} CBTC, interference measurements, channel sounding, GNSS

\section{INTRODUCTION}

Today both public and individual transport are challenged by the increasing urbanization causing congestion, delays, and personal inconveniences. The transportation systems of the future address these negative impacts by becoming increasingly digitized and networked, i.e. truly C-ITS. C-ITS will increase transport capacity and safety while reducing the environmental impact. In road transport, several wireless communication technologies have been standardized and are competing for market shares in the $5.9 \mathrm{GHz}$ ITS band for C-ITS, e.g. European Telecommunications Standards Institute (ETSI) ITS operating in the $5 \mathrm{GHz}$ frequency band (ITS-G5) [1], 3rd Generation Partnership Project - Long-Term Evolution (3GPP-LTE) cellular V2X (C-V2X) [2], fifth generation (5G) new radio (NR) V2X [3], Institute of Electrical and Electronics Engineers (IEEE) 802.11bd next generation V2X (NGV) [4].

At the same time, urban rail operators have been deploying the communication-based train control (CBTC) for regional railways and subways in cities in the $5.9 \mathrm{GHz}$ ITS band [5]. At a first glance, the spatial separation of road C-ITS and subways would certainly allow for band sharing. However, a more comprehensive analysis shows that in many cities subway lines also run above ground and often in close vicinity to roads. Thus, there is only a slight spatial separation between the radio technologies for road and rail C-ITS in urban environments. Without appropriate measures, interference between the different radio technologies must be assumed and reliable communication for safety-critical applications cannot be guaranteed [5].

In order to develop appropriate measures, accurate and realistic interference models for the current and future radio technologies are needed. Hence, we conducted an interference measurement campaign with ITS-G5, C-V2X, and 802.11a as desired train to ground (T2G) link between a base station (BS) and the DB advanced TrainLab and two infering car to train (C2T) links in Berlin on the "Süd-Ring" during four days. In parallel, we measured the receiver power for both $\mathrm{C} 2 \mathrm{~T}$ links and the channel transfer function for one $\mathrm{C} 2 \mathrm{~T}$ link allowing us to develop precise interference models for current and future V2X radio technologies. In the following, we present an overview on C-ITS radio technologies and the measurement campaign as well as first results and conclusions.

\section{C-ITS Radio Technologies}

In the automotive industry, the ITS-G5 communication system [1], which is based on IEEE 802.11p [6], is currently introduced throughout Europe in the $5.9 \mathrm{GHz}$ band [7]. An essential functionality of the ITS-G5 data link layer besides the medium access control (MAC) is the decentralized congestion control (DCC) that controls the network load reducing packet collisions, losses, and delays in high traffic density scenarios. In parallel, 3GPP has standardized in Release 14 LTE C-V2X communications [8] ]. 3GPP-LTE C-V2X has been adapted by ETSI as alternative access technology for C-ITS besides ITS-G5 [9]. Both 3GPP and IEEE continue to drive standardization for $\mathrm{V} 2 \mathrm{X}$ radio communications. In the case of 3GPP, C-V2X has been updated in Release 15 [10] and a new 5G-NR-V2X technology has been developed within the 3GPP standardization in Release 16 and 17 [3], [11]. The IEEE 802.11 Working Group is developing an $802.11 \mathrm{p}$ backwards compatible standard within the NGV Project $802.11 \mathrm{bd}$ [4]. In the following, all current and future radio technologies for 
V2X, i.e. ITS-G5, LTE C-V2X, 5G-NR-V2X and NGV, are summarized under the term V2X communications technology.

Parallel to V2X communications technology for C-ITS in road traffic, train control systems with wireless communications are being introduced and further developed in railway operations in Europe. For high-speed lines between cities, the European Train Control System (ETCS) is used in international and national rail traffic [12]. This system uses the 20 year old Global System for Mobile Communications (GSM) for Railways (GSM-R) standard for wireless communication between train and infrastructure. Currently, the International Union of Railways (UIC), 3GPP, and ETSI are working on replacing GSM-R with the Future Railway Mobile Communications System (FRMCS) [13], [14], which will be based most likely on 3GPP's $5 \mathrm{G}$ technology.

In urban areas, CBTC is used especially for regional railways and subways [15]. There are no mandatory communication technologies standardized for CBTC [5]. Many commercial implementations use wireless local area network (WLAN) radio systems according to the IEEE 802.11 standard [16] in the license-free Industrial, Scientific and Medical (ISM) bands or in licensed bands. Since the ISM bands are used by the public for internet access and consumer electronics, there is often a lot of interference in these bands. Thus, reliable wireless communication based on WLAN is impossible.

Due to the scarcity of the frequency bands, licenses for spectrum use are often very expensive, especially if harmonized use across a country or Europe is sought. For this reason, the regulatory authorities are seeking multiple use of bands where the spatial separation of radio applications allows the frequency reuse without significant interference. At first glance, the spatial separation would certainly be the case for C-ITS in road traffic and subways. In a more comprehensive analysis, however, it becomes clear that in many cities subway lines also run above ground and thus there is only a slight spatial separation between the radio technologies for road and rail C-ITS in urban environments. Without appropriate measures, interference in radio communication between the systems must be assumed and reliable communication for safety-critical applications cannot be guaranteed.

\section{MEASUREMENT CAMPAIGN}

To that end, we planned, prepared and executed a four-day measurement campaign in the project Vehicle-to-Everything Radio for digital, urban Train Communications (V2X-DuRail) [17], which involved a train and two cars in different urban scenarios, such as the one depicted in Fig. 1. After evaluating different options, we decided to conduct the measurements in the area of the "Südring" of the Berlin Ringbahn [18]. Each scenario is characterized by the environmental elements, the vehicle maneuvers, and their speeds. Regarding the environmental elements, we consider tunnels or cross-bridges as characterizing factors besides other elements like buildings and vegetation, which are present in any urban scenario. Regarding the maneuvers, we consider three main ones: train and cars driving in the same direction, driving in the opposite direction,

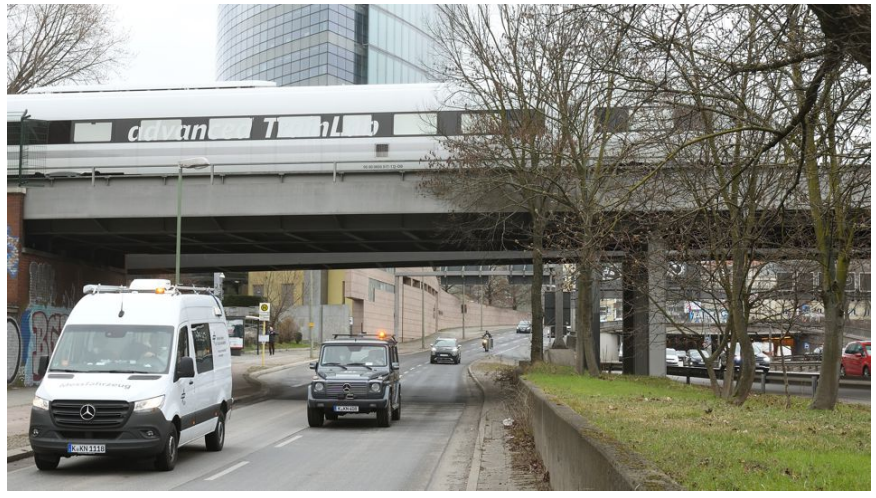

Fig. 1. V2X-DuRail measurements with DB Advanced Trainlab and two measurement cars of DLR in Berlin

TABLE I

MEASUREMEnt SCEnARIO PARAMETERS

\begin{tabular}{|c|c|c|c|}
\hline Option & 1 & 2 & 3 \\
\hline Area & $\begin{array}{c}\text { Tempelhof }- \\
\text { Neukölln }\end{array}$ & $\begin{array}{c}\text { Tempelhof - } \\
\text { Grunewald }\end{array}$ & - \\
\hline Maneuver & $\begin{array}{c}\text { parallel } \\
\text { along }\end{array}$ & crossing & $\begin{array}{c}\text { parallel } \\
\text { opposite }\end{array}$ \\
\hline Route & LOS & NLOS & - \\
\hline T2G link & $802.11 \mathrm{a}$ & ITS-G5 & C-V2X \\
\hline Interference & no & $\begin{array}{c}\text { adjacent } \\
\text { channel }\end{array}$ & inband \\
\hline
\end{tabular}

or crossing at a bridge or under pass (cf. Fig. 11). Finally, the speed of the vehicles was chosen to fulfill the requirements of the V2X and localization equipment.

\section{A. Scenarios and maneuvers}

To execute the measurements, we defined specific scenarios with parameters as listed in Tab. If First, we conducted the measurement in two different areas of Berlin. Area 1 covered mainly an urban environment and Area 2 a suburban environment. Next, we selected different driving maneuvers (see Tab. I) and different route options with LOS or non-LOS (NLOS) between the cars and train. Finally we selected the communications technology for the $\mathrm{T} 2 \mathrm{G}$ link and possible interference settings. Out of the possible 108 options we selected 51 that could be executed given the train schedule.

\section{B. Measurement equipment and setup}

The general setup for this measurement campaign was one T2G and two C2T communication links. The units at the BS and the cars were setup as transmitter (Tx), the units installed in the train as receiver $(\mathrm{Rx})$. The $\mathrm{T} 2 \mathrm{G}$ link emulating CBTC could be established either by ITS-G5, C-V2X or 802.11a wireless communication. The $\mathrm{C} 2 \mathrm{~T}$ communication used two ITS-G5 links for each car. The first link was setup to measure packet error rate (PER) and Rx power without interference and the second link was used to cause interference on the T2G link. Note that in order to obtain more measurement data at larger distances, unidirectional high power amplifiers were employed on the $\mathrm{T} 2 \mathrm{G}$ and $\mathrm{C} 2 \mathrm{~T}$ links. This practically disabled the MAC or DCC of the corresponding $\mathrm{V} 2 \mathrm{X}$ radios. To gain 
TABLE II

OVERVIEW COMMUNICATION LINKS

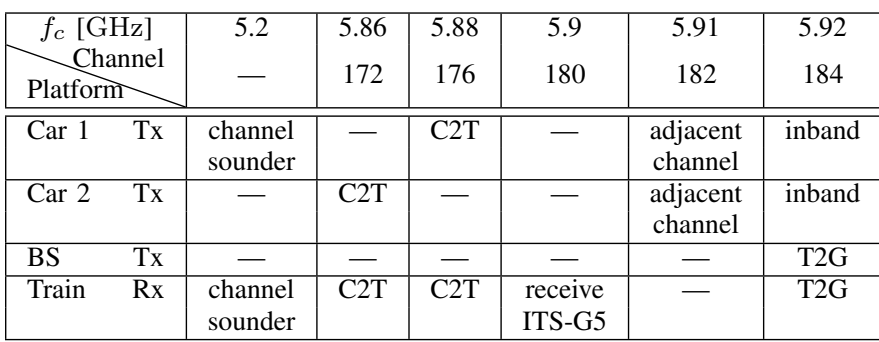

more insights on the propagation for the $\mathrm{C} 2 \mathrm{~T}$ communication, channel sounder measurements were performed in parallel between Car 1 and the train. The whole setup results in several communication links using the frequency spectrum as listed in Table II

1) Platforms: The three measurement vehicles in Fig. 1 are described in the sequel together with the BS for the T2G link:

- The BS for the T2G link was built up on a tripod and placed on a bridge over the railway track. The T2G communication could be switched between ITS-G5, $\mathrm{C}-\mathrm{V} 2 \mathrm{X}$ and 802.11a. The signal was radiated from a $18 \mathrm{dBi}$ gain directional antenna pointing eastwards to the railway track resulting in maximum Tx power of $39 \mathrm{dBm}$. The position of the BS was tracked with a Septentrio PolaRx5 TR global navigation satellite system (GNSS) receiver.

- Car 1 was a Mercedes van with omni-directional antennas mounted on a $2 \mathrm{~m} \times 1 \mathrm{~m}$ antenna platform in $3.5 \mathrm{~m}$ height. Car 1 was used as Tx for C2T ITS-G5 link, as interferer on the T2G link, and for the channel sounder link. The position was tracked with a Septentrio PolaRx5 and an u-blox F9R GNSS receiver.

- Car 2 was a Mercedes sports utility vehicle (SUV) equipped with an ITS-G5 Tx and an omni-directional antenna at $2 \mathrm{~m}$ height transmitting on the C2T link and interfering on the T2G link. The position was tracked with a Septentrio PolaRx5 TR and an u-blox F9R GNSS receiver.

- The DB advanced TrainLab was the main measurement vehicle. All installed equipment in the train was setup as receiver. We installed four ITS-G5, one C-V2X, one 802.11a, and one channel sounder receiver to capture data from the T2G and $\mathrm{C} 2 \mathrm{~T}$ links. On a unique antenna platform on top of the train (cf. Fig. 2), three train certified antennas were installed:

1) An $8 \mathrm{dBi}$ gain, omni-directional Huber+Suhner SWA-0859/360/4/0/DFRX30 antenna,

2) A $10 \mathrm{dBi}$ gain, 4-port Antonics OmPlecs-TOP 200 AMR MF-06-4 antenna,

3) A $9 \mathrm{dBi}$ gain, omni-directional Antonics OmPlecsTOP 200 AMR 1500 B L1/L2 H GNSS antenna.

Whereas the channel sounder could be electronically switched between the omni-directional Huber+Suhner

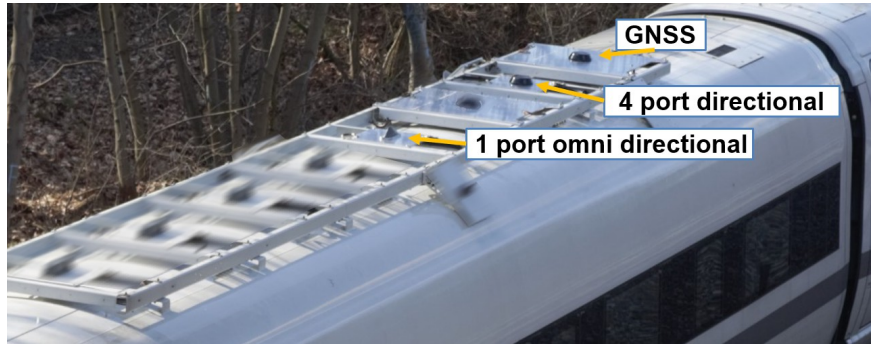

Fig. 2. Antennas installed on DB Advanced Trainlab

antenna and the 4-port antenna, all ITS-G5, C-V2X, and 802.11a receiver were connected only to the omnidirectional antenna. To compensate subsequent cable and splitter losses, a $20 \mathrm{~dB}$ gain Mini-Circuits ZX60-83LN$\mathrm{S}+$ low-noise amplifier (LNA) was directly connected to the antenna output. The GNSS antenna supported the position tracking with a Septentrio PolaRx5 receiver.

2) IEEE 802.11a: In order to emulate CBTC according to [5] with state-of-the art communications technology, we chose to transmit 802.11a compliant packets on Channel 184 with a $5 \mathrm{MHz}$ bandwidth. For that we employed an ETTUS Research Universal Software Radio Peripheral B210 software defined radio (SDR) together with the "IEEE $802.11 \mathrm{a} / \mathrm{g} / \mathrm{p}$ transceiver for GNU Radio" software [19]. The SDR transmitted 564 byte long packets with binary phase shift keying (BPSK) $R=1 / 2$ at a rate of $32 \mathrm{~Hz}$ resulting in a $10 \%$ channel busy ratio (CBR). According to [5], this CBR corresponds to an average channel occupancy for CBTC. At the train we used a corresponding B210 SDR to capture received packets and measure PER and Rx power.

3) LTE C-V2X: First, we chose C-V2X as alternative to the 802.11a T2G link. For that we used a Cohda MK6 evaluation kit. Due to some implementation issues, the MK6 transmitted 100 byte long packets with quaternary PSK $R=1 / 4$ at $10 \mathrm{~Hz}$ causing a CBR of $3 \%$ to $6 \%$.

4) ITS-G5: As second alternative to the 802.11a T2G link, we used a Cohda MK5 road side unit transmitting ITS-G5 at the BS. The MK5 was set to transmit 564 byte long packets BPSK $R=1 / 2$ at a rate of $64 \mathrm{~Hz}$ in the $10 \mathrm{MHz}$ channel 184 . This caused a $10 \%$ CBR similar to 802.11a.

For the C2T link, Cohda MK5 on-board units (OBUs) were used in both the train and the cars. Each car was equipped with one MK5 OBU set up in Tx mode. One of the radios of the MK5 in each of the cars was tuned on an individual channel (172 or 176) while the second radio was tuned in both cars to act as inband or adjacent-channel interference on the $\mathrm{T} 2 \mathrm{G}$ link. On these channels the vehicles transmitted 100, 600, and 1200 byte long packets with BPSK $R=1 / 2$ at a sum rate of $300 \mathrm{~Hz}$. This resulted in a channel occupancy of about $50 \%$ per car. On Car 1 each radio output was connected to a high power amplifier and to a $6 \mathrm{dBi}$ Huber+Suhner omni-directional antenna placed on a metal plate on the roof resulting in a maximum Tx power of $36 \mathrm{dBm}$. On Car 2 both radio outputs were connected through an RF-combiner and a power amplifier 
to a $9 \mathrm{dBi}$ ECOM antenna on the roof resulting in a maximum Tx power of $35 \mathrm{dBm}$.

At the train, we employed 4 Cohda MK5 OBUs to receive the $\mathrm{C} 2 \mathrm{~T}$ and the T2G links as well as to monitor the ITS-G5 Control Channel 180 (cf. Tab. III).

5) Channel Sounder: The propagation measurements were performed with the RUSK Medav-DLR channel sounder [20]. For all measurements the operating center frequency was set to $5.2 \mathrm{GHz}$ with a bandwidth of $120 \mathrm{MHz}$. The emitted output power was $46 \mathrm{dBm}$ and the transmitted signal was similar to an orthogonal frequency-division multiplexing (OFDM) signal. The receiver unit recorded the channel transfer function for each snapshot. The measurement length was fixed to $6.4 \mu \mathrm{s}$. The channel sounder was set up either in single-input singleoutput (SISO) or in single input, multiple output (SIMO) operation. The snapshot rate was set to $488 \mathrm{~Hz}$ or $244 \mathrm{~Hz}$ for SISO or SIMO. For SISO, the receiver was connected to the omni-directional Huber+Suhner antenna, whereas for SIMO, the receiver was switching between the 4 ports of the 4-port Antonics antenna.

6) Localization: A set of various localization sensors were installed on the train to support the measurements. For the interference campaign the most relevant were:

- GNSS was used at both the train and the cars as the primary source of absolute geographic location information. All vehicles were equipped with a Septentrio PolarRx5 (TR) multi-frequency, multi-constellation GNSS receiver. The cars were additionally equipped with an u-blox F9R multi-frequency, multi-constellation GNSS receiver featuring an inertial measurement unit. These receivers obtained real-time GNSS corrections over the German SAPOS service and computed an inertial-aided phasefixed solution in real-time. In this way, a decimeterlevel position solution at a rate of $5 \mathrm{~Hz}$, along with its orientation and velocity, was obtained for both cars.

- Laserscanner: As an additional source of position information and in order to further stabilize the GNSS solution, each of the cars was equipped with a Velodyne VLP-16 laser scanner on its roof. The VLP-16 is a 16 plane, $360^{\circ}$ laser scanner, which is able to scan points up to a distance of $120 \mathrm{~m}$. The laser scanner allows in postprocessing to locate the car relatively to its environment by applying a simultaneous localization and mapping method. The resulting point map can additionally be used, along with the video footage, to get further information on the environment and draw conclusions on the propagation conditions of $\mathrm{C} 2 \mathrm{~T}$ communication.

\section{DATA ANALYSis}

In the sequel we analyze the measurement data for the scenario in the Tempelhof - Neukölln area with parallel along driving maneuver, mainly a LOS route between the cars and the train, ITS-G5 as T2G link, and adjacent channel interference from the cars. Fig. 4 shows a top view of the scenario and driven routes of the vehicles. The BS is located at $(0 \mathrm{~m}, 0 \mathrm{~m}))^{1}$ in the local east-north-up (ENU) coordinate system. The train drives from Tempelhof in the west entering the map at $(-480 \mathrm{~m},-80 \mathrm{~m})$ and continues towards Neukölln at $(1390 \mathrm{~m}, 320 \mathrm{~m})$. While the train approaches, the cars start to drive slowly from the bridge, where the BS is located, into the street parallel to the railway tracks. Here the car antennas are between $5 \mathrm{~m}$ and $6.6 \mathrm{~m}$ above the train antenna. Note that Fig. 3 only shows the Rx and noise power for successfully received packets.

Examining the plots in Fig. 3, we can distinguish five zones:

1) Before $13512 \mathrm{~s}$ at a distance of greater than $217 \mathrm{~m}$, the train receives no data from the $\mathrm{BS}$ as it is out of coverage due to the directional antenna.

2) Between $13512 \mathrm{~s}$ and $13605 \mathrm{~s}$ the interference from the cars is negligible. In particular between $13567 \mathrm{~s}$ and 13604 s no packet errors were measured. As can be seen from Fig. $3 b$, the estimated $\mathrm{Rx}$ power in this region is much higher than the estimated noise power including interference.

3) From $13605 \mathrm{~s}$ to $13738 \mathrm{~s}$, the $\mathrm{T} 2 \mathrm{G}$ link experiences severe interference from the adjacent channel with PERs ranging from $0.8 \%$ to $53 \%$. Note that the worst case interference is depicted in Fig. 3 by the black vertical line and in Fig. 4 by the enlarged markers. Clearly, Fig. $3 \mathrm{~b}$ shows the increased estimated noise power due to interference of the successfully received packets. Comparing the Rx power in Fig. 3c with the one in Fig. 3b. the C2T links have now a similar or higher Rx power than the T2G link. Taking into account the spectral mask of ITS-G5 Tx [1], the interference power in the adjacent channel should be at least $26 \mathrm{~dB}$ lower than the $\mathrm{Rx}$ power in Fig. 3c Nevertheless, this interference level is sufficient to cause at least $39 \%$ PER.

4) From $13738 \mathrm{~s}$ to $13833 \mathrm{~s}$ the PER drops below $8.7 \%$ and in some cases even to zero. On a first sight this is counter intuitive as the distance between the cars and the train is smaller than the one between the BS and the train (cf. Fig. 3a). Examining Fig. 3c and Fig. 4 , one can determine that the interfering $\mathrm{C} 2 \mathrm{~T}$ links experience significant power drops due to shadowing from a bridge and stairs to a train platform at $(650 \mathrm{~m}, 146 \mathrm{~m})$ whereas the T2G link is still LOS.

5) After $13834 \mathrm{~s}$ the PER increases again as the train moves out of coverage of the T2G link. This observation is confirmed by the drop in Rx power in Fig. $3 \mathrm{~b}$ below $90 \mathrm{~dB}$. Please note, Car 1 is turning then into a parallel road with buildings blocking the LOS between the C2T link. The distance between Car1 and the train ranges from $272 \mathrm{~m}$ to $794 \mathrm{~m}$, but no packets are received and no $\mathrm{Rx}$ power on the C2T link can be measured.

\section{CONCLUSION}

To summarize, we conducted a four-day interference measurement campaign with the DB advanced TrainLab and two

\footnotetext{
${ }^{1}$ For brevity of notation, we omit the up-coordinate.
} 


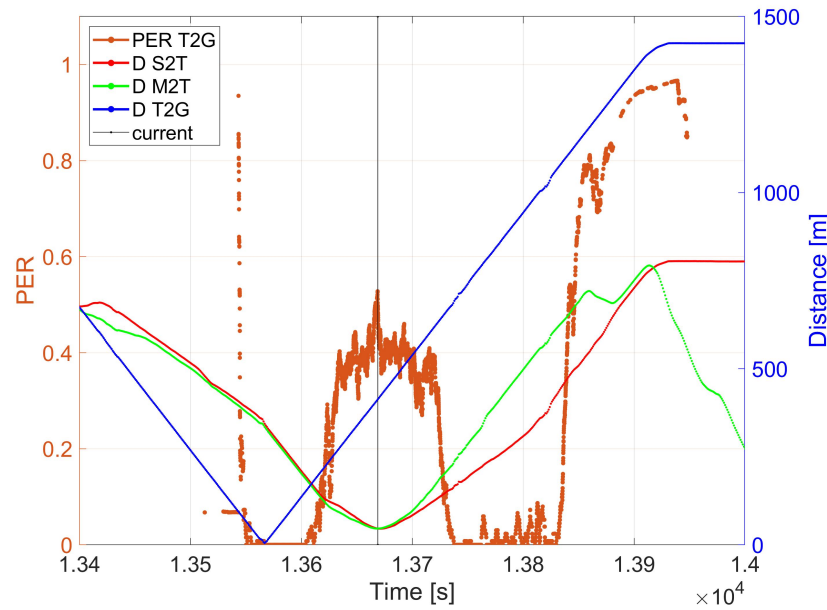

(a) PER and Distance (D)

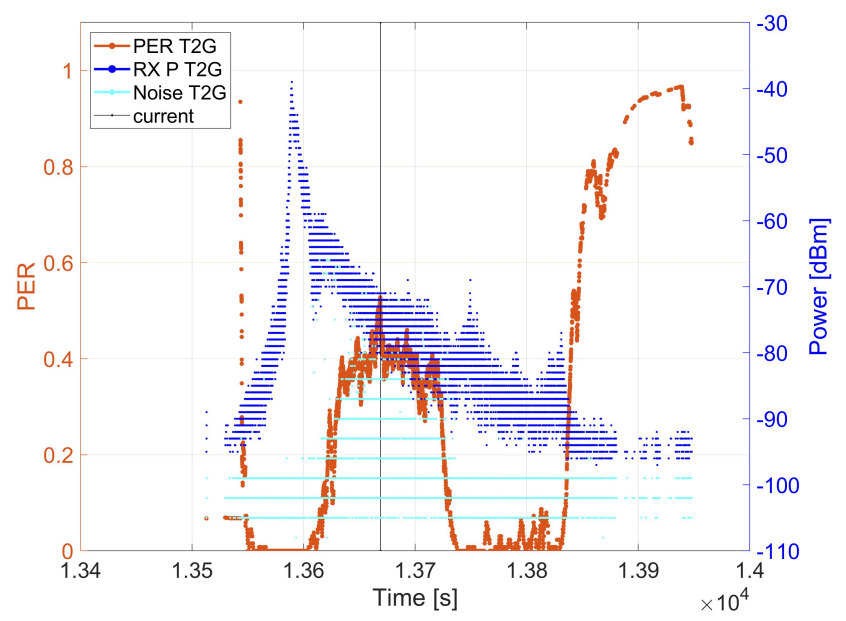

(b) PER and RX / Noise Power for T2G link

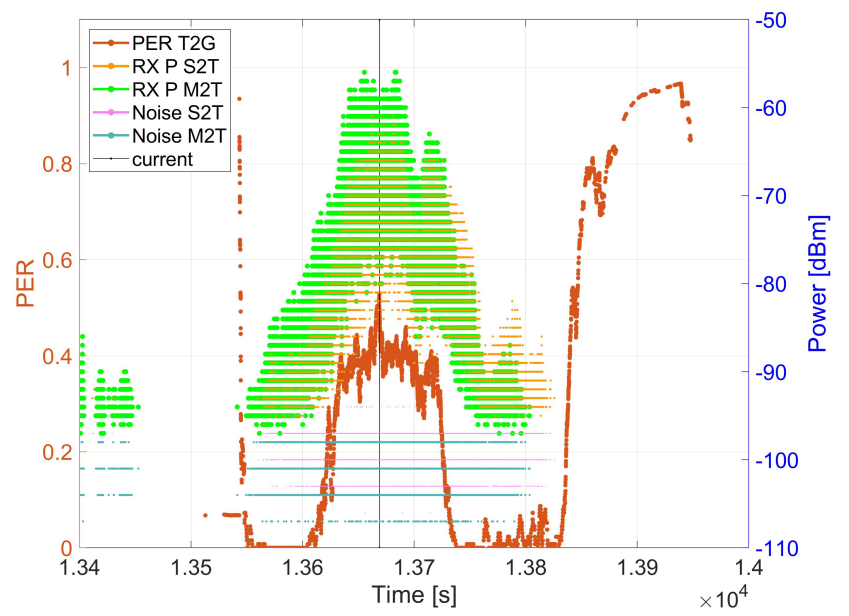

(c) PER and RX / Noise Power (P) for C2T link

Fig. 3. PER, Distance, and RX / Noise Power (P) versus time for T2G and C2T links: Train (T), Car 1 (measurement van (M)), Car 2 (SUV (S)), and $\mathrm{BS}$ (G) road cars in the city of Berlin to assess the interference between urban road and rail traffic in the same or adjacent channels. Simultaneously, we measured the Rx power on each C2T link without interference and for Car 1 the wide band channel transfer function. The first results show that even adjacent channel interference, where the spectral mask of ITS-G5 should at least lower the interference power by $26 \mathrm{~dB}$, causes a severe increase in PER to more than $39 \%$ for an ITS-G5 T2G link in a LOS scenario with high interference load. Note that the high interference load was obtained by disabling the MAC and DCC of the corresponding V2X radios. We conclude from the scenario analyzed so far, that a relatively small protection zone of about $200 \mathrm{~m}$ radius in LOS to the train could protect the T2G link from interfering V2X communication of road C-ITS. With MAC and DCC enabled, we expect that the protection zone could be further reduced.

In our future work, we will analyze the presented and the remaining 50 scenarios in depth for the different radio technologies. In addition, we will develop novel interference models based on the channel measurements and derive measures to protect the interference victim. Furthermore, we will make some of the measurement data publicly available through the V2X-DuRail website [17] to foster scientific collaboration with interested researchers.

\section{ACKNOWLEDGMENT}

The authors would like to express their deep gratitude to the DB Advanced TrainLab team enabling this complex measurement campaign. Likewise, we would like to thank the team from Intelligence on Wheels (IoW) for supporting the coordination of all the maneuvers with their protection technology. Further thanks go to Maschinenbau und Service GmbH Ammendorf and Carsten Ribbecke (DLR) helping us to mount measurement equipment in and on the train. The presented work is in parts carried out in the V2X-DuRail project funded by the German Ministry of Transport and Digital Infrastructure through the mFUND initiative under the grant VB18F1048A [17] and in the Intelligent Magnetic Positioning for Avoiding Collisions of Trains (IMPACT) project funded by the Bavarian Ministry of Economic Affairs, Regional Development and Energy under the grant DIK-2002-0016 / DIK0175/01 [21].

\section{REFERENCES}

[1] ETSI, "Intelligent Transport Systems (ITS); ITS-G5 Access layer specification for Intelligent Transport Systems operating in the $5 \mathrm{GHz}$ frequency band," ETSI EN 302663 V1.3.1, pp. 1-25, Jan. 2020.

[2] 3GPP, "Initial Cellular V2X standard completed," Sep. 2016, accessed: May 14, 2021. [Online]. Available: https://www.3gpp.org/news-events/ 3gpp-news/1798-v2x_r14

[3] - "Technical Specification Group Services and System Aspects; Release 16 Description; Summary of Rel-16 Work Items (Release 16)," 3GPP TR 21.965 V1.0.0, Dec. 2020.

[4] IEEE P802.11 Task Group BD, "P802.11bd - Enhancements for Next Generation V2X," IEEE P802.11 Task Group BD (NGV), Dec. 2018. [Online]. Available: https://www.ieee802.org/11/Reports/tgbd_update. $\mathrm{htm}$

[5] ETSI, "Urban Rail ITS and Road ITS applications in the 5,9 GHz band; Investigations for the shared use of spectrum," ETSI TR 103580 V1.1.1, pp. 1-118, Aug. 2019. 


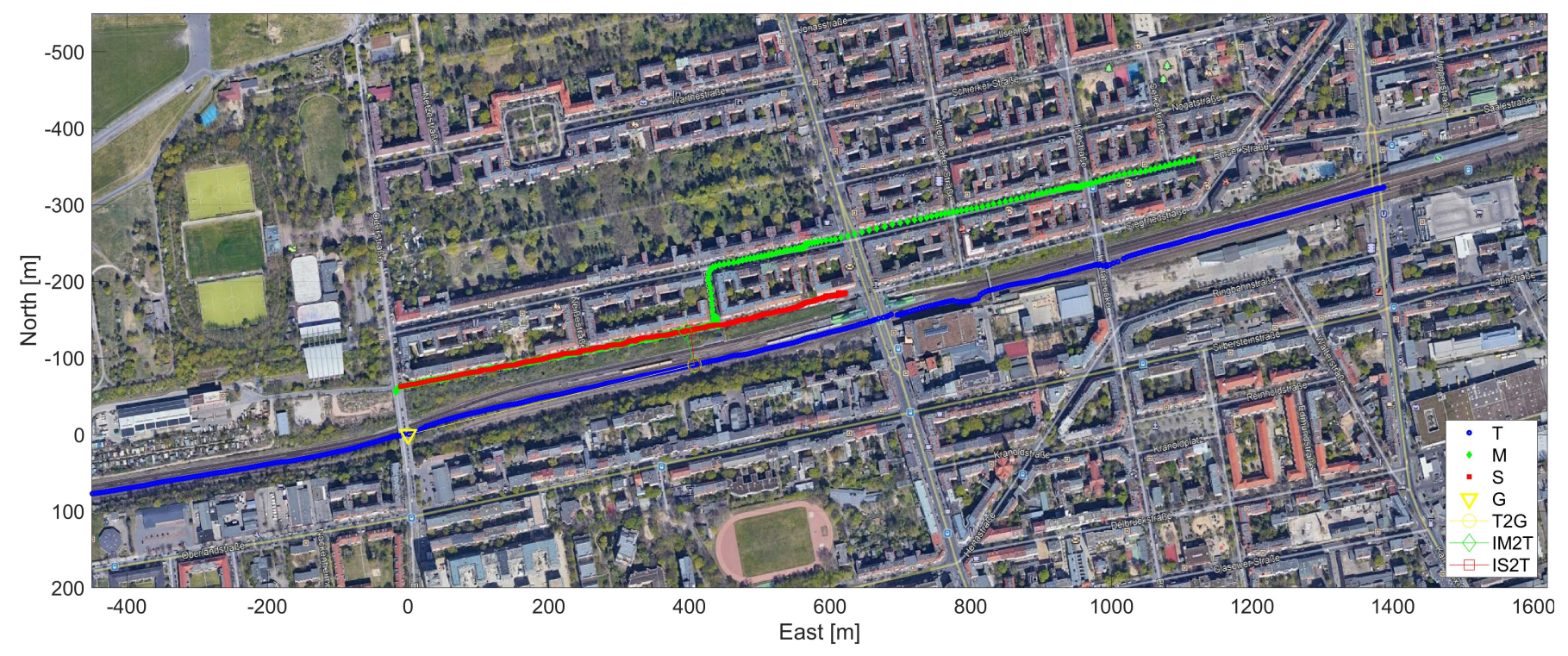

Fig. 4. Positions of train (T), Car 1 (measurement van (M)), Car 2 (SUV (S)), and BS (G) on a map between Tempelhof in the west and Neukölln in the northeast. Solid lines show geometric LOS T2G, interfering (I) M2T and S2T links. Map data: (O2021 Google, O2021 GeoBasis-DE/BKG

[6] IEEE, "IEEE Standard for Information technology- Local and metropolitan area networks- Specific requirements- Part 11: Wireless LAN Medium Access Control (MAC) and Physical Layer (PHY) Specifications Amendment 6: Wireless Access in Vehicular Environments," IEEE Std 802.11p-2010, pp. 1-51, 2010.

[7] Volkswagen, "Car2x in the new golf: A 'technological milestone'," accessed: May 14, 2021. [Online]. Available: https://www.volkswagen-newsroom.com/en/stories/ car2x-in-the-new-golf-a-technological-milestone-5919

[8] 3GPP, "Technical Specification Group Services and System Aspects; Release 14 Description; Summary of Rel-14 Work Items (Release 14)," 3GPP TR 21.914 V14.0.0, May 2018.

[9] ETSI, "Intelligent Transport Systems (ITS); LTE-V2X Access layer specification for Intelligent Transport Systems operating in the $5 \mathrm{GHz}$ frequency band," ETSI EN 303613 V1.1.1, pp. 1-20, Jan. 2020.

[10] 3GPP, "Technical Specification Group Services and System Aspects; Release 15 Description; Summary of Rel-15 Work Items (Release 15)," 3GPP TR 21.915 V15.0.0, Sep. 2019.

[11] — , "Release 17," 2020, accessed: May 14, 2021. [Online]. Available: https://www.3gpp.org/release-17

[12] ERA, "Control Command and Signalling (CCS) Technical Specifications for Interoperability (TSIs) Annex A- Mandatory Specification (ETCS and GSM-R baselines)," Aug. 2019, accessed: May 14, 2021. [Online]. Available: https://www.era.europa.eu/content/ ccs-tsi-annex-mandatory-specifications_en

[13] UIC, "Future Railway Mobile Communication System $\neg$ User Requirements Specification," FRMCS Functional Working Group, FU-7100, Version 4.0.0, pp. 1-120, Jan. 2019.

[14] ETSI, "Rail Telecommunications (RT); Future Rail Mobile Communication System (FRMCS); Study on system architecture," ETSI TR 103 459 V1.2.1, pp. 1-73, Aug. 2020.
[15] IEEE P1474 Working Group CBTC, "IEEE Recommended Practice for Communications-Based Train Control (CBTC) System Design and Functional Allocations," IEEE Std 1474.3-2008, pp. 1-129, 2008.

[16] IEEE, "IEEE Standard for Information Technology-Telecommunications and Information Exchange between Systems - Local and Metropolitan Area Networks-Specific Requirements - Part 11: Wireless LAN Medium Access Control (MAC) and Physical Layer (PHY) Specifications," IEEE Std 802.11-2020, pp. 1-4379, 2021.

[17] DLR, "V2X-DuRail - Vehicle-to-Everything Radio for digital urban train communication," 2021, accessed: May 14, 2021. [Online]. Available: https://www.dlr.de/kn/en/desktopdefault.aspx/ tabid-4308/6940_read-70030/admin-1/

[18] Wikipedia contributors, "Berlin ringbahn - Wikipedia, the free encyclopedia," 2021, accessed: May 14, 2021. [Online]. Available: https://en. wikipedia.org/w/index.php?title=Berlin_Ringbahn\&oldid=1014656840

[19] B. Bloessl, M. Segata, C. Sommer, and F. Dressler, "An IEEE $802.11 \mathrm{a} / \mathrm{g} / \mathrm{p}$ OFDM Receiver for GNU Radio," in ACM SIGCOMM 2013, 2nd ACM Software Radio Implementation Forum (SRIF 2013). Hong Kong, China: ACM, Aug. 2013, pp. 9-16.

[20] P. Unterhuber, S. Pfletschinger, S. Sand et al., "A Survey of Channel Measurements and Models for Current and Future Railway Communication Systems," Mobile Information Systems, vol. 2016, pp. 1-14, 2016. [Online]. Available: http://www.hindawi.com/journals/ misy/2016/7308604/

[21] DLR, "IMPACT - Intelligent Magnetic Positioning for Avoiding Collisions of Trains," 2021, accessed: May 14, 2021. [Online]. Available: https://www.dlr.de/kn/desktopdefault.aspx/tabid-2081/6941_ read-72013/admin-1/ 